\title{
DIELECTRIC PROPERTIES OF TRIGLYCINE SULPHATE CRYSTALS ADMIXTURED WITH L-THREONINE
}

\author{
A. Czarnecka, J. Stankowska and S. Mielcarek \\ Institute of Physics, A. Mickiewicz University \\ Grunwaldzka 6, 60-780 Poznań, Poland \\ (Received January 18, 1994; in final form March 8, 1994)
}

\begin{abstract}
The influence of L-threonine admixture on the dielectric properties of triglycine sulphate (TGS) crystals was studied. With increasing concentration of the admixture in the solution from which the crystals were grown, their maximum permittivity and spontaneous polarization were found to decrease, their Curie point was found not much different than that for pure TGS crystals (322 K), the coercive field and bias field increased. The domain structure of the obtained crystals was also studied by the liquid crystal method.
\end{abstract}

PACS numbers: 77.22.Ch

\section{Introduction}

Introduction of organic admixtures into TGS crystals causes fixation of one of the polarization senses: $+P_{\mathrm{s}}$ or $-P_{\mathrm{s}}$ which leads to the appearance of a bias field, $E_{\mathrm{b}}$, in the crystal [1]. Owing to this phenomenon dielectric parameters of the admixtured crystals undergo only slight changes in the process of spontaneous ageing, which is of particular importance in the application of TGS crystals. So far the influence of such admixtures as L- and D-alanine [1-5], L-, D-, DL-serine [6-8], L-valine [12], phosphaglycine [9] and haloacetamide [10] on physical properties of TGS crystals has been studied. This paper presents results of investigation of L-threonine admixture on dielectric properties of TGS crystals (LTTGS). Part of the results was presented at the 8 IMF in USA [13]. Both L-threonine and glycine, which is a component of TGS crystals, are aminoacids. Glycine of the chemical formula $\mathrm{CH}_{2}\left(\mathrm{NH}_{2}\right) \mathrm{COOH}$ contains in its alkyl group only one functional group $\mathrm{NH}_{2}$ whereas threonine $\mathrm{CH}_{3} \mathrm{CH}(\mathrm{OHI}) \mathrm{CH}\left(\mathrm{NH}_{2}\right) \mathrm{COOH}$ besides $\mathrm{NH}_{2}$ also contains $\mathrm{OH}$ functional group. This difference is responsible for different reactivities of glycine and threonine.

\section{Experimental}

LTTGS crystals were grown from aqueous solutions containing 5, 10, 15 and $20 \mathrm{wt} \%$ of L-threonine relative to TGS mass. The crystals were grown at a constant temperature either in ferroelectric or in paraelectric phase. Samples to be studied were cut out from the (001) or (110) growth pyramids. The sample surfaces perpendicular to their ferroelectric axis were covered by evaporated silver 
electrodes. LTTGS crystals grow along the "b" axis asymmetrically with respect to the nucleus, similarly as it has been observed for LATGS [3, 4], DATGS [5], LSTGS [6] and DSTGS [7] crystals.

Measurements of permittivity were done using an RLC bridge of the measuring field frequency $1 \mathrm{kHz}$. Dielectric hysteresis loops were measured by a Diamant Drenck Pepinsky system. The domain structure of the crystals was studied by liquid crystal method [11] (MBBA) under polarization microscope.

\section{Results}

LTTGS crystals grow asymmetrically with respect to the $b$ axis. The rate of the crystal growth in the direction $+b$ is greater than in $-b$. Already for low concentrations of admixture the crystal growth in the $-b$ direction was very small. Morphology of LTTGS crystals is also different than that of TGS crystals. The former grow large (121) walls of which in TGS crystals only residues are observed, in LTTGS crystals the walls (110) do not grow and the walls type the (111) and (100) grow only for low concentrations of the admixture.

Single crystals grown from solutions containing $20 \%$ of $L$-threonine admixture reveal small increase in the direction of the $c$ axis (the crystal is plane). Temperature measurements of permittivity, $\varepsilon(T)$, were made upon cooling and heating for aged and young TGS crystals admixtured with different amounts of L-threonine. Rejuvenation of the samples was performed by annealing them at about $353 \mathrm{~K}$ for 24 hours.

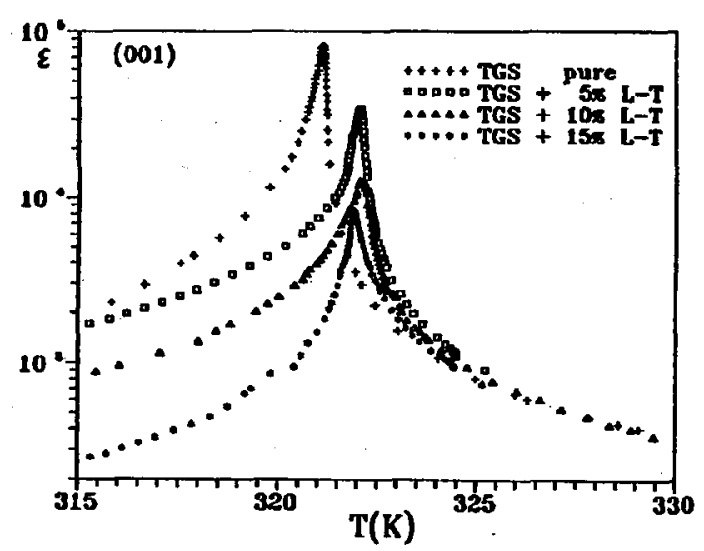

Fig. 1. Temperature dependence of permittivity for rejuvenated L-threonine admixtured TGS crystals grown below $T_{\mathrm{c}}$ (cooling).

Figure 1 presents the temperature dependence of permittivity obtained for young samples of LTTGS crystals grown below the Curie temperature, cut out from (001) growth pyramid of the longer part of the crystal ( $+b$ direction).

With increasing concentration of $\mathbf{L}$-threonine in the solution from which a given crystal was grown, the permittivity $\varepsilon_{\max }$ as well as $\Delta \varepsilon_{\max }$ defined as 
$\Delta \varepsilon_{\max }=\varepsilon_{\max }$ (cooling) $-\varepsilon_{\max }$ (heating), were found to decrease. The same kind of dependence was observed for both growth pyramids and for crystals grown in ferro- as well as in paraelectric phase. The Curie temperatures of the studied crystals oscillate about $322 \mathrm{~K}$ taking values differing from it by $-0.2 \mathrm{~K}$ or $+0.5 \mathrm{~K}$, and it was difficult to find any regularity which could be related to the influence of $\mathrm{L}$-threonine admixture in TGS crystals. The values of $\varepsilon_{\max }, \Delta \varepsilon_{\max }, T_{\mathrm{c}}$ and $\Delta T_{\mathrm{c}}$ obtained for all studied samples are collected in Table I.

TABLE I

The values of maximum permittivity $\varepsilon_{\max }, \Delta \varepsilon_{\max }$, and the values of the Curie temperatures $T_{\mathrm{c}}$ and $\Delta T_{\mathrm{c}}$ in various growth pyramids for young and aged LTTGS crystals with different content of L-threonine admixture for samples cut out from crystals grown below and above the Curie point.

\begin{tabular}{|c|c|c|c|c|c|c|}
\hline \multicolumn{7}{|c|}{ Growth pyramid (001), $T_{\mathrm{g}}<T_{\mathrm{c}}$} \\
\hline \multirow{2}{*}{$\begin{array}{l}\text { wg } \% \text { of } \\
\text { L-threonine } \\
\text { in solution }\end{array}$} & \multicolumn{2}{|c|}{$\begin{array}{l}T_{\mathrm{c}} \\
{[\mathrm{K}]}\end{array}$} & \multirow[t]{2}{*}{$\begin{array}{l}\Delta T_{\mathrm{c}} \\
{[\mathrm{K}]}\end{array}$} & \multicolumn{2}{|c|}{$\begin{array}{l}\varepsilon_{\max } \\
\left(10^{3}\right)\end{array}$} & \multirow[t]{2}{*}{$\begin{array}{r}\Delta \varepsilon_{\text {max }} \\
\left(10^{3}\right)\end{array}$} \\
\hline & aged & young & & aged & young & \\
\hline 0 & 322.3 & 321.1 & 1.2 & 12.8 & 82.4 & 69.6 \\
\hline 5 & 321.5 & 322.1 & -0.6 & 17.5 & 34.9 & 17.4 \\
\hline 10 & 322.2 & 322.1 & 0.1 & 9.6 & 13.0 & 3.4 \\
\hline 15 & 322.2 & 321.9 & 0.3 & 10.8 & 8.5 & -2.3 \\
\hline 20 & 321.9 & 322.2 & -0.3 & 4.7 & 5.8 & 1.1 \\
\hline \multicolumn{7}{|c|}{ Growth pyramid $(110), T_{\mathrm{g}}<T_{\mathrm{c}}$} \\
\hline $\mathbf{0}$ & 322.1 & 321.9 & 0.2 & 10.5 & 80.0 & 69.5 \\
\hline 5 & 321.4 & 322.0 & -0.6 & 22.4 & 43.9 & 21.5 \\
\hline 10 & 321.8 & 321.4 & 0.4 & 11.8 & 13.9 & 2.1 \\
\hline 15 & 320.5 & 321.3 & -0.8 & 2.6 & 3.5 & 0.9 \\
\hline 20 & 321.7 & 321.5 & 0.2 & 4.2 & 5.5 & 1.3 \\
\hline \multicolumn{7}{|c|}{ Growth pyramid (001), $T_{\mathrm{g}}>T_{\mathrm{c}}$} \\
\hline $\mathbf{0}$ & 322.0 & 321.9 & 0.1 & 32.7 & 122.0 & 89.3 \\
\hline 5 & 321.9 & 322.0 & -0.1 & 15.1 & 35.6 & 20.5 \\
\hline 10 & 321.6 & 322.0 & -0.4 & 11.0 & 38.5 & 27.5 \\
\hline 15 & 320.5 & 320.3 & 0.2 & 3.1 & 3.7 & 0.6 \\
\hline \multicolumn{7}{|c|}{ Growth pyramid (110), $T_{\mathrm{g}}>T_{\mathrm{c}}$} \\
\hline 0 & 321.8 & 322.0 & -0.2 & 21.1 & 69.1 & 48.0 \\
\hline 5 & 321.8 & 321.9 & -0.1 & 7.8 & 20.2 & 12.4 \\
\hline 10 & 321.5 & 322.0 & -0.5 & 16.0 & 18.8 & 2.8 \\
\hline 15 & 322.1 & 322.0 & -0.1 & 6.5 & 11.1 & 4.6 \\
\hline
\end{tabular}



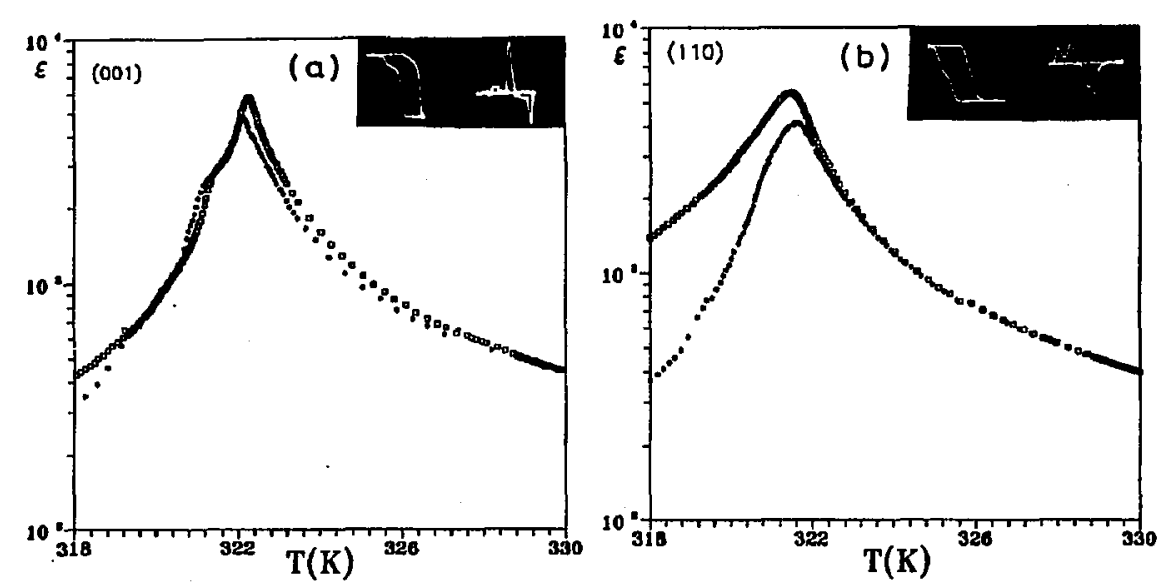

Fig. 2. Temperature dependencies of permittivity for aged and rejuvenated TGS + $20 \%$ L-threonine samples cut out from (a) (001) and (b) (110) growth pyramids. * heating, $\square-$ cooling. The top right corners show dielectric hysteresis and current loops.

The most interesting results were found for crystals grown from the solutions containing $20 \%$ of L-threonine. Figure 2 presents temperature dependencies of permittivity for samples cut out from the (001) (Fig. 2a) and (110) (Fig. 2b) growth pyramids. The curve shows broadening of the phase transition (Fig. 2b) and a change in the slope of $\varepsilon(T)$ dependence near $T_{\mathrm{c}}$ from the ferroelectric side for a sample cut out from (001) growth pyramid (Fig. 2a).

Dielectric hysteresis loops of the samples cut out from (001) and (110) growth pyramids have been found to be narrow and asymmetric, see the top right corner in Fig. 2.

The bias field of these crystals was determined to be $558 \times 10^{2} \mathrm{~V} / \mathrm{m}$ for samples cut out from (001) growth pyramid and $239 \times 10^{2} \mathrm{~V} / \mathrm{m}$ for samples cut out from (110) one.

As it was established earlier [14], more admixture is built into the slower growing part of the crystal, i.e. from the $-b$ side, than in the faster growing part. Regarding this fact we studied a sample cut out from the slower growing side of the crystal of TGS $+15 \%$ L-threonine, i.e. from the $-b$ side. As the crystal growth from this side is really very small, the sample is not cut out from a single growth pyramid.

For this sample we measured the temperature dependence of permittivity and dielectric hysteresis loop at room temperature. Figure 3 presents the obtained dependencies of $\varepsilon(T)$ for an aged sample (Fig. 3a) and a sample rejuvenated for 3 days (Fig. $3 \mathrm{~b}$ ). The dependence reveals a double peak which does not disappear after a long time annealing of the sample at a temperature above $T_{\mathrm{c}}$. The hysteresis loops both for aged and rejuvenated sample are irregular which is especially well visible for current loops, Fig. 3 (the top right corner in Fig. 3).

Temperature dependencies of spontaneous polarization, $P_{\mathbf{s}}$, coercive field, $E_{\mathrm{c}}$, and bias field, $E_{\mathrm{b}}$, were studied for samples including L-threonine admixture 

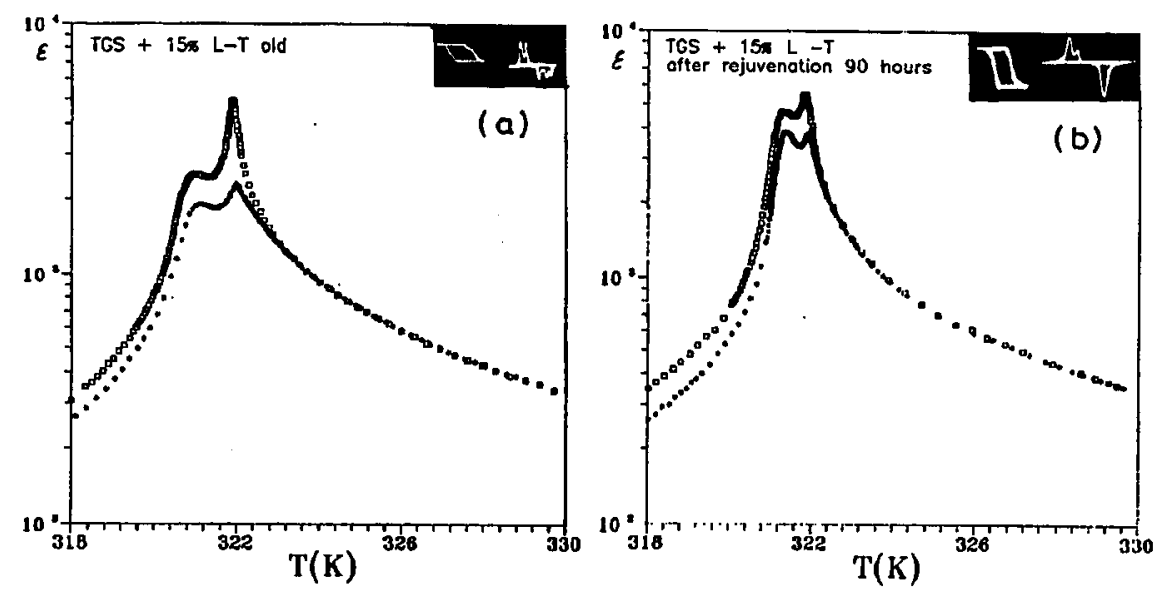

Fig. 3. Temperature dependencies of permittivity for aged (a) and rejuvenated for 90 hours (b) TGS $+15 \%$ L-threonine sample cut out from the $-b$ side of the crystal. The top right corners show dielectric hysteresis and current loops. Photographs of the loops were taken for different oscilloscope multiplication $U_{y}$; (a) $0.5 \mathrm{~V} / \mathrm{cm}$, (b) $0.2 \mathrm{~V} / \mathrm{cm}$. * - heating, $\square-$ cooling.
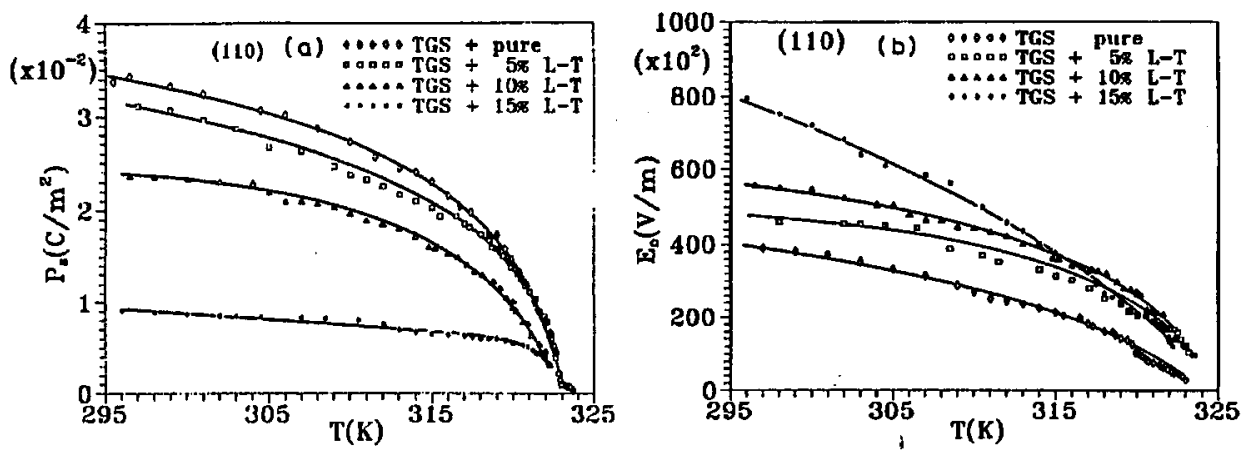

Fig. 4. Temperature dependence of $P_{s}(\mathrm{a})$ and $E_{c}$ (b) for LTTGS crystals grown below the Curie point.

at different concentrations. Figure $4 \mathrm{a}$ and $4 \mathrm{~b}$ presents the results obtained for young samples cut out from TGS crystals admixtured with different amount of L-threonine grown in ferroelectric phase.

Table II collects the values of spontaneous polarization, coercive field and bias field obtained for all crystals studied. As follows from the obtained curves and Table II with increasing concentration of the admixture in the solution from which a studied crystal was grown, the values of $P_{\mathrm{s}}$ decrease while $E_{\mathrm{c}}$ increases. Moreover, investigation of dielectric hysteresis loops has shown that irrespective of the growth pyramid, $P_{\mathrm{s}}$ takes lower values and $E_{\mathrm{c}}$ higher ones for crystals grown in ferroelectric phase than for those grown in paraelectric phase. 
TABLE II The values of the dielectric hysteresis loop parameters for aged and rejuvenated L-threonine admixtured TGS cut out from different growth pyramids.

\begin{tabular}{|c|c|c|c|c|c|c|}
\hline \multirow{2}{*}{$\begin{array}{l}\text { wg \% of } \\
\text { L-threonine } \\
\text { in solution }\end{array}$} & \multicolumn{2}{|c|}{$\begin{array}{c}P_{\mathrm{s}} \\
{\left[\mu \mathrm{C} / \mathrm{cm}^{2}\right]}\end{array}$} & \multicolumn{2}{|c|}{$\begin{array}{c}E_{\mathrm{c}} \\
{[\mathrm{V} / \mathrm{cm}]}\end{array}$} & \multicolumn{2}{|c|}{$\begin{array}{c}E_{\mathrm{b}} \\
{[\mathrm{V} / \mathrm{cm}]}\end{array}$} \\
\hline & old & young & old & young & old & young \\
\hline \multicolumn{7}{|c|}{ Growth pyramid $(001), T_{\mathrm{g}}<T_{\mathrm{c}}$} \\
\hline 0 & 2.2 & 2.8 & 440 & 500 & 110 & 160 \\
\hline 5 & 3.5 & 2.9 & 600 & 540 & 100 & 200 \\
\hline 10 & 2.7 & 2.1 & 560 & 590 & 530 & 400 \\
\hline 15 & 2.9 & 2.2 & 570 & 620 & 1600 & 800 \\
\hline \multicolumn{7}{|c|}{ Growth pyramid (110), $T_{\mathrm{g}}<T_{\mathrm{c}}$} \\
\hline 0 & 2.9 & 3.2 & 660 & 410 & 100 & 50 \\
\hline 5 & 3.4 & 3.0 & 850 & 460 & 640 & 300 \\
\hline 10 & 3.0 & 2.4 & 680 & 560 & 2300 & 500 \\
\hline 15 & 1.7 & 0.9 & 1100 & 840 & 7100 & 2100 \\
\hline \multicolumn{7}{|c|}{ Growth pyramid $(001), T_{\mathrm{g}}>T_{\mathrm{c}}$} \\
\hline 0 & 3.2 & 3.1 & 410 & 380 & 80 & 40 \\
\hline 5 & 2.5 & 2.7 & 520 & 460 & 120 & 80 \\
\hline 10 & 3.3 & 2.9 & 1300 & 470 & 230 & 120 \\
\hline 15 & 2.7 & 1.7 & 6500 & 710 & 300 & 260 \\
\hline \multicolumn{7}{|c|}{ Growth pyramid $(110), T_{\mathrm{g}}>T_{\mathrm{c}}$} \\
\hline 0 & 2.5 & 2.4 & 550 & 420 & 150 & 160 \\
\hline 5 & 2.7 & 2.4 & 300 & 480 & 100 & 150 \\
\hline 10 & 3.2 & 2.8 & 470 & 490 & 200 & 200 \\
\hline 15 & 3.0 & 2.5 & 520 & 610 & 700 & 430 \\
\hline
\end{tabular}

Comparing the values of $P_{\mathrm{s}}$ and $E_{\mathrm{c}}$ for samples cut out from different growth pyramids we find that for crystals grown above the Curie point these values do not differ much whereas for crystals grown below $T_{\mathrm{c}}$ the values of $P_{\mathrm{s}}$ are lower and $E_{\mathrm{c}}$ higher for (001) growth pyramid than for (110) one. The crystals grown from the solutions of TGS $+20 \%$ L-threonine are characterized by distorted hysteresis loops. Long-time annealing of the samples at $350 \mathrm{~K}$ did not lead to symmetrization of the loops. Applying different values of the measuring field we could observe participation of subsequent regions of fixed domains in the process of repolarization. The occurrence of fixed regions was confirmed by the character of the temperature dependence of these samples permittivity. In the $\varepsilon(T)$ curve, on the ferroelectric side (Fig. 2a), we could distinguish two slopes. 
Domain structure of LTTGS crystals was studied by the liquid crystal method. We examined aged and young TGS crystals admixtured with different amounts of L-threonine, grown either below or above the Curie point. The crystals grown in ferroelectric phase included domains of larger size and more regular shape than those grown in paraelectric phase. In aged crystals the domains were of irregular shapes and frayed edges. Rejuvenation of TGS crystals containing a low concentration of L-threonine results in refinement of their domain structure and in the shapes of their domains becoming similar to the shapes of domains in pure TGS crystal. Crystals with higher admixture of L-threonine despite annealing above $T_{\mathrm{c}}$, reveal a fixed domain structure of large irregular domains. Changes in domain structure during spontaneous ageing of LTTGS crystals grown above and below the Curie point were also examined. Figure 5 presents photographs of domain structure and Fig. 6 - the dependence of the number of domains per $1 \mathrm{~mm}$ of the crystal length on logarithm of time, obtained for TGS $+5 \%$ of L- threonine.

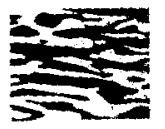

$2.5 \mathrm{~h}$

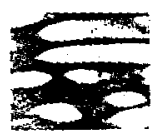

$2.5 \mathrm{~h}$

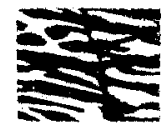

$8 \mathrm{~h}$

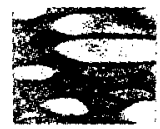

$3 \mathrm{~h}$

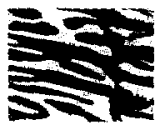

$26 \mathrm{~h}$

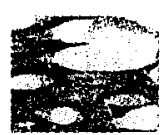

$4 \mathrm{~h}$

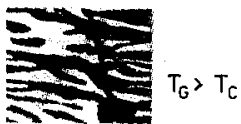

$54 \mathrm{~h}$

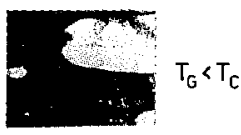

$7 \mathrm{~h}$

Fig. 5. Photographs of domain structure in the process of spontaneous ageing taken at different times for TGS $+5 \%$ L-threonine crystals grown above and below the Curie point.

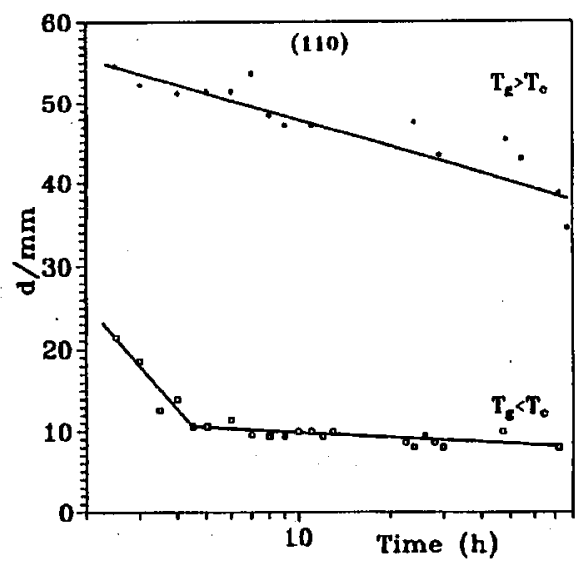

Fig. 6. The number of domains per $1 \mathrm{~mm}$ of the crystal length vs. the time that has elapsed since the sample passed through the Curie point, for the crystals of TGS $+5 \%$ of L-threonine. 

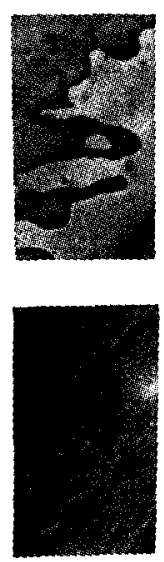

$3 h$
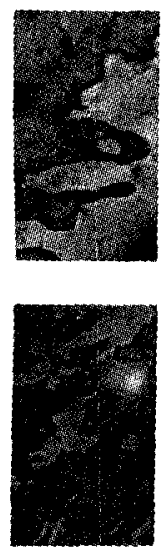

$4 h$

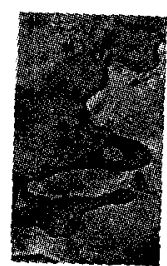

a.

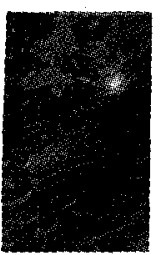

$26 \mathrm{~h}$

Fig. 7. Photographs of domain structure in the process of spontaneous ageing for TGS $+20 \%$ L-threonine crystals observed for (110) growth pyramid: (a) crystal grown below $T_{\mathrm{c}}$, (b) crystal grown above $T_{\mathrm{c}}$.

For the crystals grown below $T_{c}$ the domains are of the shape of separate lenses while for those grown above $T_{\mathrm{c}}$ the lenses are joint forming irregular bands, Fig. 5 . The constants, $S$, characterizing the rate of spontaneous ageing of TGS crystals admixtured with $5 \%$ of $\mathrm{L}$-threonine were determined as tangents of the curves in Fig. 6. Spontaneous ageing of crystals grown below the Curie point occurs in two stages. In the beginning the shape of domains and their number per $1 \mathrm{~mm}$ of the crystal length change very fast, at a rate constant $S=47.5$ domains $/(\mathrm{mm} \mathrm{h})$, after about 6 hours the rate of these changes slows down to $S=1.85$ domains $/(\mathrm{mm}$ h). For crystals grown above the Curie point, the changes of the shape of domains and their number per $1 \mathrm{~mm}$ are slow in the whole time range $(2.2-80 \mathrm{~h})$ considered $S=9.54$ domains $/(\mathrm{mm} \mathrm{h})$.

Domain structures of TGS crystals admixtured with $20 \%$ of L-threonine grown below and above the Curie point were also studied. In rejuvenated crystals, grown in ferro- and paraelectric phase, we observed large areas of fixed domains within the volume of the whole sample. Figure 7 presents photographs of the domain structure observed in the process of spontaneous ageing in the (110) growth pyramid in the crystals grown below $T_{c}$ (Fig. $7 \mathrm{a}$ ) and above $T_{\mathrm{c}}(7 \mathrm{~b}$ ). As can be seen this structure changes very little upon ageing.

\section{Discussion}

In all studied TGS crystals admixtured with L-threonine we find the presence of bias field, $E_{\mathrm{b}}$, whose intensity depends on concentration of L-threonine in solutions from which the crystals have been grown. Figure 8 presents the dependence of $E_{\mathrm{b}}$ on L-threonine concentration in solution obtained for young samples.

We have found that the $E_{\mathrm{b}}$ intensity increases with increasing concentration of L-threonine in solution from which the studied crystals were grown, both for 


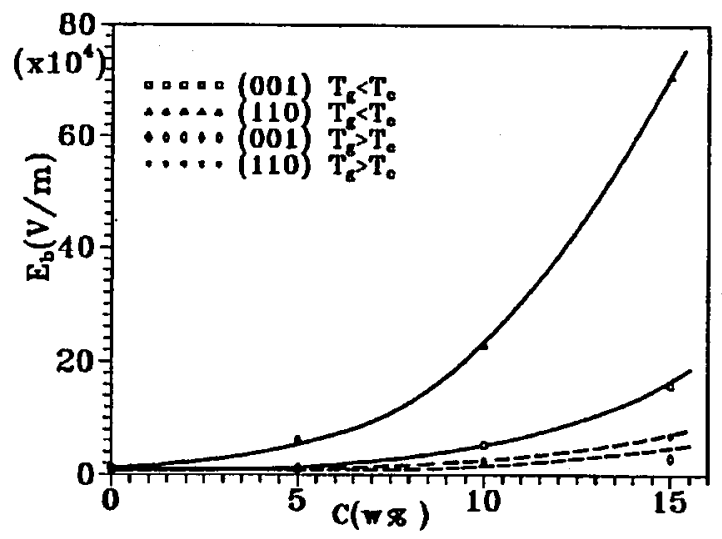

Fig. 8. The bias field $E_{\mathrm{b}}$ vs. concentration of $\mathrm{L}$-threonine admixture in TGS solution for LTTGS crystals grown below and above the Curie point in two (001) and (110) growth pyramids.
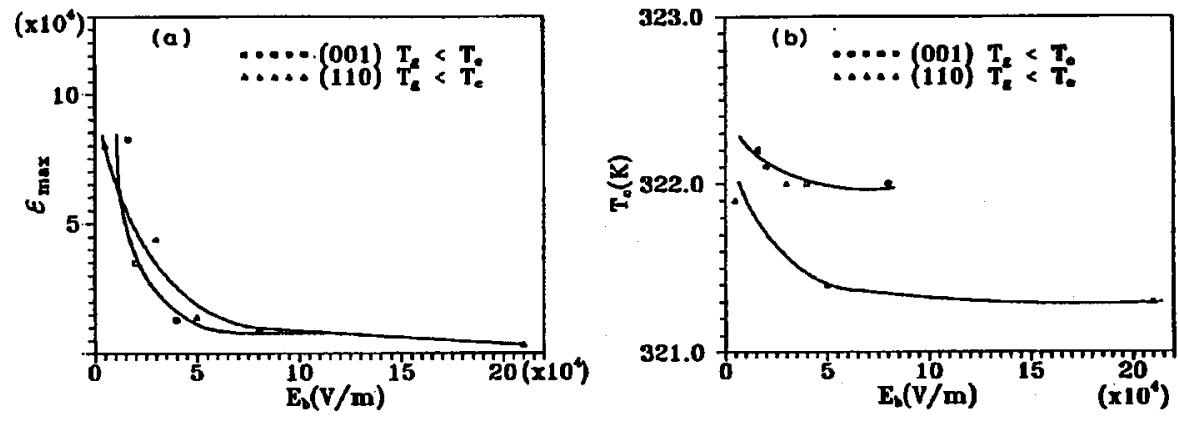

Fig. 9. The dependence of maximum permittivity $\varepsilon_{\max }$ (a) and the Curie temperature $T_{\mathrm{c}}$ (b) on bias field $E_{\mathrm{b}}$ of LTTGS crystals grown in ferroelectric phase.

young and aged crystals. The values of $E_{\mathrm{b}}$ are higher for the crystals grown below $T_{\mathrm{c}}$ than for those grown above it. Intensity of $E_{\mathrm{b}}$ can be treated as a measure of concentration of the admixture in the crystal. Figure 9 presents dependencies of the maximum permittivity (Fig. 9a) and the Curie temperature $T_{\mathrm{c}}$ (Fig. 9b) on the bias field value, for LTTGS crystals. $\varepsilon_{\max }$ and $T_{c}$ exponentially decrease with increasing $E_{\mathrm{b}}$ both for the (001) and (110) growth pyramids.

The character of these dependencies can be explained by assuming that the bias field acts in the same way as an external electric field applied to the crystal. Thus the higher the $E_{\mathrm{b}}$ the lower the $\varepsilon_{\max }$. The bias field also affects $P_{\mathrm{s}}$ and $E_{\mathrm{c}}$. The former decreases whereas the latter increases with increasing $\boldsymbol{E}_{\mathbf{b}}$.

Our interpretation of the double peak observed in Fig. 3 in $\varepsilon(T)$ dependence for sample cut out from the $-b$ side of the crystal is that the sample was cut out from the region including the boundaries of the (121) and (110) growth pyramids and the existing domain structure was particularly strongly fixed on defects. The 
fixation is so strong that it is not destroyed even when the sample is annealed at a temperature above $T_{\mathrm{c}}$. The sample comprises regions of a different number of ferroelectrically active dipoles and it is known that the Curie temperature is a linear function of the number of ferroelectrically active dipoles. Therefore, a spatial distribution of lattice defect concentration entails spatial distribution of the Curie temperature and this may be the reason why we observe a double peak in the $\varepsilon(T)$ dependence.

In the process of crystal growth L-threonine admixture is built into the crystal lattice of TGS crystals replacing probably one of glycines. Glycines I-III are planar in the paraelectric phase while in ferroelectric phase glycine I becomes nonplanar. When glycine $I$ is replaced by $L$-threonine molecule, $P_{s}$ becomes fixed and bias field, which modifies all the measured dielectric parameters of the crystal, appears. The replacement of glycine I by L-threonine occurs more frequently when the crystal is grown in ferroelectric phase. Changes in dielectric parameters caused by introducing L-threonine admixture are greater than those following the introduction of L-valine [12] which is a consequence of different structures of L-threonine and L-valine.

\section{References}

[1] K.L. Bye, P.W. Whips, E.T. Keve, Ferroelectrics 4, 235 (1972).

[2] M: Koralewski, J. Stankowska, T. Jasiniski, Jpn. J. Appl. Phys. 26, 383 (1987).

[3] N. Nakatani, Jpn. J. Appl. Phys. 30, 1024 (1991).

[4] B. Brezina, M. Havrankova, Cryst. Res. Technol. 20, 787 (1985).

[5] B. Brezina, M. Havrankova, Cryst. Res. Technol. 20, 781 (1985).

[6] J. Stankowska, A. Czarnecka, G. Kwitowska, Ferroelectrics 108, 1931 (1990).

[7] J. Stankowska, A. Czarnecka, L. Dejneka, Ferroelectrics 124, 55 (1991).

[8] J. Stankowska, A. Czarnecka, A. Dratwińska, Ferroelectrics 140, 65 (1993).

[9] S. Mielcarek, J. Stankowska, Ferroelectrics 81, 95 (1988).

[10] S. Mielcarek, J. Stankowska, Acta Phys. Pol. A 72, 691 (1987).

[11] V.P. Konstantinova, N.A. Tikhomirova, M. Glogarova, Ferroelectrics 20, 259 (1978).

[12] J. Stankowska, S. Mielcarek, A. Czarnecka, M. Musial, in: Abstracts - The Eighth International Meeting on Ferroelectricity, Maryland (USA), 1993, Ferroelectrics, in press.

[13] J. Stankowska, A. Czarnecka, S. Mielcarek, K. Słabolepsza, in: Abstracts - The Eighth International Meeting on Ferroelectricity, Maryland (USA), 1993, Ferroelectrics, in press.

[14] J. Stankowska, A. Czarnecka, Bangobango Dongo Gemi, Acta Phys. Pol. A 83, 485 (1993). 